\title{
Method of Medvedkov V. D. for the destruction of coronavirus
}

\author{
Victor Medvedkov* \\ Gzhel State University, 140155, Gzhel, Russia
}

\begin{abstract}
The human cell is one of the best places to implement the main goal of the SARSCoV-2 coronavirus-reproduction. Due to its small size from 80 to $220 \mathrm{~nm}$, it is invisible, highly contagious and pervasive. Full protection from it is possible only with the help of maximum self-isolation. It is impossible when working in society and even when living in a family, i.e. the majority of the world's population will be infected with it. Plus from infectionthe development of herd immunity to the new coronavirus; cons-loss of part of the alveoli, leading to hypoxia of the body, disruption of the brain and many organs and parts of the individual's body, sometimes to a cytokine storm and death. To protect the human body from the COVID-19 coronavirus infection, a biologically based, tested, and accessible method is proposed. It consists on the rational use of heat factors and hot alcohol vapors that do not reduce the immune system. The experiment took place on the 66-year-old World champion, 3-time European champion,12-time Russian champion and was successful.
\end{abstract}

\section{Introduction}

The SARS-CoV-19 coronavirus has found the best place for it to live and reproduce - humans and intends to stay with it for millennia. In this regard, each person needs to be armed with a simple, cheap, affordable way for each individual to effectively destroy the recently appeared killer-the new coronavirus on the body and inside it.

Traumatization, immobilization, and destruction of the new coronavirus on and inside the body is most effective with alcohol, temperature, and UV exposure [1-6]. The numerous anti-coronavirus medications and vaccines offered, as well as the method described below, require careful additional verification.

$70 \%$ alcohol breaks the fat envelope of the coronavirus, making it inoperable for movement and reproduction. However, the high concentration of alcohol in the human body lowers its immunity $(7,8)$ and, accordingly, the ability to protect it from the new coronavirus. Temperature exposure to the coronavirus above 56 degrees, according to well-known virologists, also immobilizes it and stops its reproduction; at 92 degrees and above, according to French scientists, this virus killer is effectively destroyed.

Strong ultraviolet exposure destroys the coronavirus in 15-30 seconds; soft ultraviolet radiation not only immobilizes the coronavirus and accelerates its destruction, but also increases the production of the body's (most useful for humans) vitamin D. It is important for improving immunity, and improves the absorption of vital calcium by the body's biological media.

The world coronavirus pandemic has activated not only virologists, but also doctors, biologists and scientists of many other scientific specialties in the search for ways to prevent and treat COVID - 19 coronavirus infection.

\section{Results and discussion}

The General idea of our method of protecting a person from COVID-19 coronavirus infection is to effectively unload his body from cells infected with SARS - CoV - 2 coronaviruses and from extracellular viruses and prevent this cytokine storm, followed by finishing off the remaining infected cells and coronaviruses by the immune system and highly effective removal of millions of dead and living viruses and infected cells from the body's biological environment with their final operational burning, that is, complete destruction of these new population killers.

The main effect of unloading the human respiratory tract and lungs from coronaviruses is based on the creation of artificial coughing or sneezing. In it millions of coronaviruses with a size of $80-220$ nanometers are thrown out of the body with their rapid burning. Artificial cough is caused by a powerful hyperventilation inhalation of vapors of just boiled 70-degree medical alcohol with a volume of up to 0.5 liters (pour it at least to the bottom of the electric kettle). The kettle we drop down the belly down so much (usually to the abdomen), to hot slightly burning

\footnotetext{
*Corresponding author: professormvd@ yandex.ru
} 
alcohol when hypervodka not injured, that is not burned, the mucous membrane of the respiratory tract and the alveoli of the lungs. Hot alcohol vapors destroy, injure, and paralyze coronaviruses and infected cells with two factors:

1) heat exposure (air temperature over 56 degrees according to virologists-traumatic for viruses, and 92 degrees according to French scientists-destroying completely new coronaviruses);

2) alcohol factor (vapors of boiled alcohol in the respiratory vessels and lungs condense, i.e. they turn from vaporous to liquid alcohol with a higher degree. It kills viruses).

Cough, as a protective reaction of the body to the necessarily non-traumatic burning of the mucous membrane, should be heard immediately after hyperventilation. The mucous membrane and healthy alveoli that are not infected with coronaviruses should be protected from burning as much as possible, since V. D. Medvedkov's loss of smell and taste before July 1, 2020 may have occurred not only due to coronaviruses, but also, possibly, due to burning of the mucosa. The high speed of exhaled air when coughing contributes to the capture and separation of millions of coronaviruses from the mucous membrane of the respiratory tract and alveoli of the lungs and their release from the body.

Released by coughing (coronaviruses fly out through the mouth) or sneezing (they are released through the nose), the bodies of invisible viruses fly to a distance of 4-6 meters, forming a large cloud of coronaviruses. We discovered this by accident using low-intensity ultraviolet light-irradiation: the subject Medvedkov V. D. at the beginning of the self-experiment coughed in the corner of the study, and the computer screen and window located to the side were splattered with coronaviruses, visible only when the ultraviolet light was turned on. Therefore, it is advisable to sneeze and cough in the steam room of the bath on a metallized foil attached above the wood-burning or electric stove of the steam bath and having a temperature of up to 300 degrees. It instantly kills coronaviruses and burns them, ensuring $100 \%$ of their disposal. This is what we did in the future.

We, the authors of the discovery "Detoxification function of physical activity" [9], strongly spurred to learn the basics of coronaviruses infection World champion, 3 times European Medvedkov V. D. coronavirus from 14 to 16 March 2020 in the championship of Russia on Sambo among masters - veterans in St.-Petersburg in sports Palace of Gazprom with impaired ventilation, or in just one battle (instead of the usual three wrestling matches) with the representative of Bashkortostan, or in the compartment of the train "Moscow - Saint-Petersburg" of the Chinese, with whom he dined at a small table compartment.

The very next day after the end of the championship, he began not aching, as usual after three veteran wrestling matches, but twisting the muscles and ligaments much stronger and longer ( 9 days instead of the usual three days) pain of the muscles of the arms and upper torso. After 3 days, physical performance began to decline sharply: on a day off, instead of the usual 10-12 hours of physical work, he could work less intensively for only 1-2 hours a day. Diarrhea lasted for 7 days. The temperature increased only by $0.5-0.7$ degrees. Gradually and imperceptibly disappeared the sense of smell and taste, which appeared only on July 1, 2020 (I remembered this day for the joy of the appearance of the taste of food for life, as it appeared suddenly on the day of the changes adoption in the Constitution of Russia).

Given that the treatment OF covid - 19 coronavirus infection in the third decade of March 2020 was carried out in hospitals with unverified (against the new SARS - CoV - 2 coronavirus) drugs and insufficiently effective technologies, we decided to develop a method for unloading the body from SARS - CoV - 2 coronaviruses, since in 1993 - 1994 we developed a patented "method for removing lead from the human body" [10], that is, we had some experience.

After the first cough, the temperature of the boiled 70-degree medical alcohol drops slightly, reducing the temperature and pressure of its vapors from the electric kettle, respectively, so we raise the latter higher and take a second hyperventilation breath, throwing out the next portion of several million coronaviruses from the body with the second cough, slightly less than with the first cough. And so we perform 4-5 coughs, effectively unloading the body from coronaviruses. This eliminates overexcitation of the immune system, protecting the body from the cytokine storm. The immune system must destroy the remaining infected cells and coronaviruses that have not been thrown out of the body.

Such 4-5-cough effects Medvedkov V. D. used 5 times a day during the onset of brain fatigue from selfeducation in coronavirusology. After the first two days of 4-5-time coughing emissions of coronaviruses from the respiratory tract and lungs, noticing with the help of ultraviolet light a high cloud spread of coronaviruses when coughing, from the third day I switched to 4-time one-day coronavirus discharge: 3 times in the office (with the release of viruses in a large cellophane bag) and once in the steam room of the bath. After 1 week, physical performance began to slowly increase and our test World champion stopped coughing off the body from coronaviruses, giving a significantly greater load to the immune system to destroy coronaviruses.

1.5 months after the coronavirus infection, V. D. Medvedkov's General and physical condition fully recovered. He is currently preparing for the World championship in Sambo among masters - veterans, which should take place in Belarus in November 2020 to check the condition of the pulmonary apparatus and the whole organism and any cost to win the fight from the representatives of the United States, whose vile policy, creating a worldwide pandemic coronavirus, taking the already economic and biogenic wars are pushing many countries to retract they started a third world war that our planet may be the last.

Features of vaping in covid - 19 coronavirus infection are as follows. Steaming is done in the same classic pattern: first the soft playing of water on the hot stones to enhance the steam and the temperature; hovering partner 
(V. D. Medvedkov when semiexperimental soared one to avoid coronavirus risk other people) two brooms back part of the legs, torso, arms; a second pair of amplification; the same soaring partner in front of the body; third pair of amplification; increased steaming back (lumbar, spine, neck). However, to increase the traumatic effect on coronaviruses, to slow down their reproduction in infected cells of the lungs and respiratory tract, it is necessary to devote $95 \%$ of the total soaring to the part of the trunk under which the lungs and respiratory tract are located after dispersing the blood flow. In addition, it is important that the person who is being steamed, while giving water to the stones by the steamer, produces a powerful hyperventilation of the lungs for additional warming of the alveoli and additional trauma of coronaviruses.

Self-experiment allowed us to draw the following conclusions:

1. Hot alcohol vapors destroy, injure, paralyze coronaviruses, contribute to a strong cough, effectively removing coronaviruses from the respiratory tract and lungs, without causing even a slight intoxication, i.e. without reducing the immune system.

2. 3-time vaping increases the removal of live and dead coronaviruses from the body that have lingered in the human biological environment with sweat (according to our calculations, 310 new giant coronaviruses are placed in one section of the small sweat duct), thereby increasing the effectiveness of the individual's anti-coronavirus protection.

\section{References}

1. I. I. Generalov, Medical Virology: textbook (VSMU, Vitebsk, 2017)

2. D. K. Lviv, R. M. Alimbarova, S. V. Alkhovsky, Medical Virology: a guide (Moscow, 2008)

3. V. M. Zhdanov, evolution of viruses (Medicine, Moscow, 1990)

4. T. I. Tikhonenko, biochemistry of viral particles (Meditsina, M., 45-87, 1977)

5. A. Rabson, A. Roit, P. Delves, Fundamentals of medical Virology (Mir, Moscow, 2006)

6. M. S. Horvitz, Virology (Mir, M., 3, 103-185, 1989)

7. A.S. Kuznetsov, Z.M. Kuznetsova, Russian Journal of Physical Education and Sport, 14(4), $5-7$ (2019). DOI: 10.14526/20704798-2019-14-4-5-7

8. V. K. Tatochenko, N. K. Ozeretskovsky, a.m. Fedorov, Immunoprophylaxis (Silver threads publishing House, Moscow)

9. V. I. Thorevsky, V. D. Medvedkov, N. I. medvedkova, Theory and practice of physical culture, 4, 24 (1997)

10. ya. I. Weissman, N. V. Zaitseva, V. D. Medvedkov, Patent for invention RU 2085178 C1, 27.07.1997 No. $94018772 / 14$ of 24.05.1994. 